
Mess- und
VDE Automatisierungstechnik
Optische Technologien
VDI-Berichte 2323

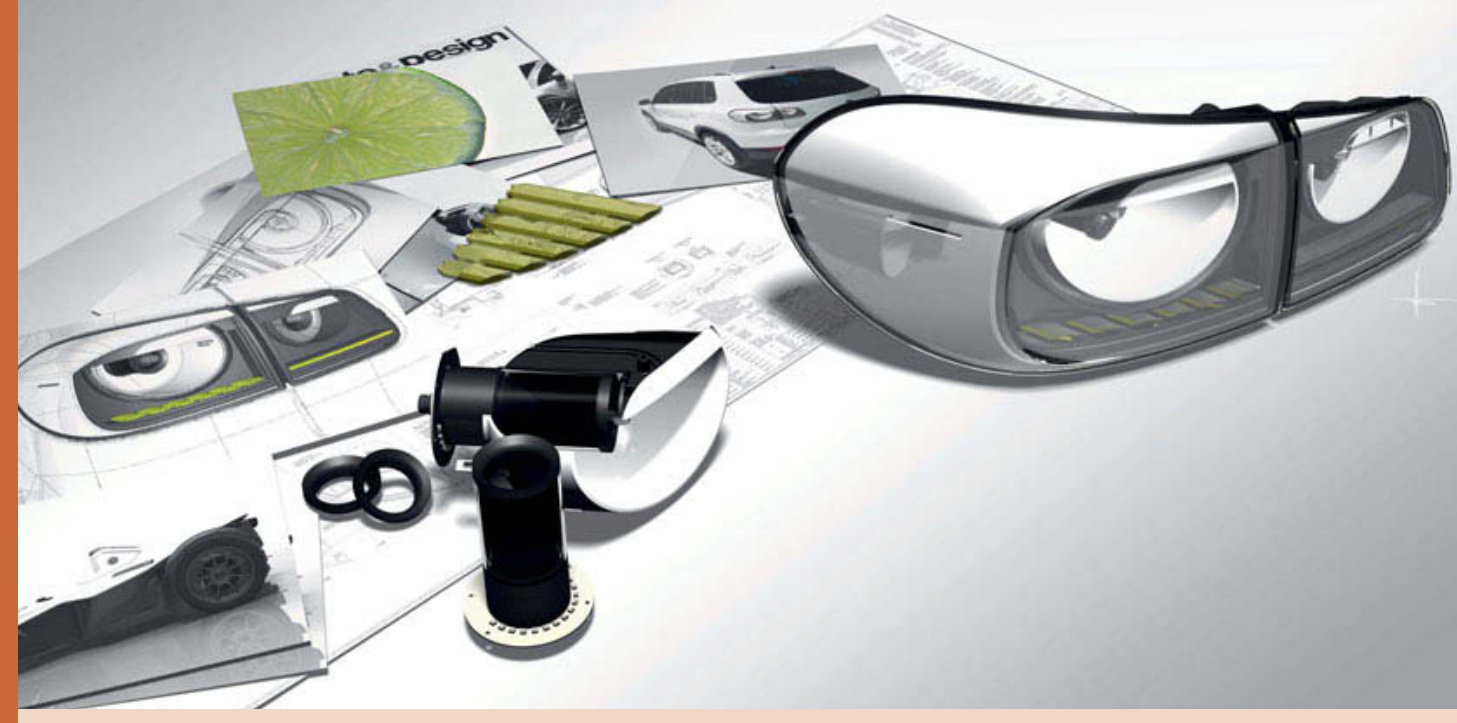

\title{
8. VDI-Fachtagung
}

Optische Technologien in der Fahrzeugtechnik

Karlsruhe, 05. und 06. Juni 2018 


\section{VDI-BERICHTE}

Herausgeber:

VDI Wissensforum GmbH 
Bibliographische Information der Deutschen Nationalbibliothek

Die Deutsche Nationalbibliothek verzeichnet diese Publikation in der Deutschen Nationalbibliographie;

detaillierte bibliographische Daten sind im Internet unter www.dnb.de abrufbar.

Bibliographic information published by the Deutsche Nationalbibliothek (German National Library)

The Deutsche Nationalbibliothek lists this publication in the Deutsche Nationalbibliographie (German National Bibliography); detailed bibliographic data is available via Internet at www.dnb.de.

\section{(c) VDI Verlag GmbH · Düsseldorf 2018}

Alle Rechte vorbehalten, auch das des Nachdruckes, der Wiedergabe (Photokopie, Mikrokopie), der Speicherung in Datenverarbeitungsanlagen und der Übersetzung, auszugsweise oder vollständig.

Der VDI-Bericht, der die Vorträge der Tagung enthält, erscheint als nichtredigierter Manuskriptdruck.

Die einzelnen Beiträge geben die auf persönlichen Erkenntnissen beruhenden Ansichten und Erfahrungen der jeweiligen Vortragenden bzw. Autoren wieder. Printed in Germany. 


\section{Inhalt}

Vorwort. . . . . . . . . . . . . . . . . . . . . .

\section{- Lichtquellen}

Benefits of Electronics Integration into LED Components $\ldots \ldots \ldots \ldots \ldots \ldots \ldots$

5. Grötsch, U. Hiller, J. Reill, R. Huber,

OSRAM Opto Semiconductors GmbH, Regensburg

Evolution und Anwendung von Automotive-LASERLichtquellen im

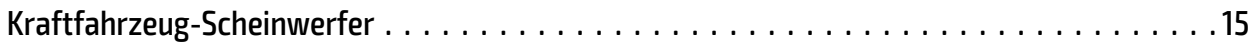
M. Licht, Automotive Lighting, Reutlingen

\section{- Hochauflösende Scheinwerfertechnologien}

Technologische und physiologische Bewertung eines hochaufgelösten

Laserscanner-Scheinwerfersystems . .

P. Ansorg, AUDI AG, Ingolstadt; Lichttechnisches Institut KIT, Karlsruhe;

M. Fischer, AUDI AG, Ingolstadt; Zentrum für Optische Technologien, Aalen University;

C. Möckel, LurexX optical GmbH, Ingolstadt;

C. Neumann, Lichttechnisches Institut KIT, Karlsruhe

Fusion zwischen Sensor- und Scheinwerfersystem - Abstandsmessung durch aktive

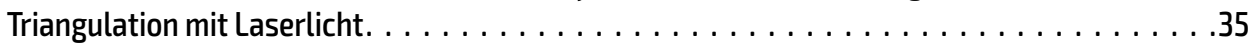

S. Mates, J. Stoll, W. Stork, Dr. Ing. h.c. F. Porsche AG, Weissach

Forschungszentrum Informatik am Karlsruher Institut für Technologie

Karlsruher Institut für Technologie, ITIV

Hochauflösender LCoS Scheinwerfer . . . . . . . . . . . . . . . . . . . . . . . . 49

J. Roth, I. Petermann-Stock, Volkswagen AG, Wolfsburg

Aktiver Scheinwerfer mit DMD-Technologie zur Erzeugung vollständiger Lichtverteilungen . . . 61 M. Knöchelmann, A. Wolf, G. Kloppenburg, R. Lachmayer,

Leibniz Universität Hannover, Institut für Produktentwicklung und Gerätebau

Digitalisierung des Lichts - Unterstützung des Fahrers in unfallträchtigen Situationen. . . . . .79 C. Wilks, B. Kubitza, HELLA GmbH \& Co. KGaA, Lippstadt 
Bewertungskriterien für die Zulassung lichtbasierter Fahrerassistenz . . . . . . . . . . 87

P. Jahn, C. Neumann, Karlsruher Institut für Technologie (KIT),

Lichttechnisches Institut (LTI), Karlsruhe

Symbolische Fahrzeug-Fußgänger-Interaktion . . . . . . . . . . . . . . . . . . .95

J. Reschke, P. Rabenau, M. Hamm, AUDI AG, Ingolstadt;

C. Neumann, Karlsruher Institut für Technologie (KIT), Karlsruhe

Tiefenwahrnehmung basierend auf aktuellen Scheinwerferentwicklungen. . . . . . . . . . 107

C. Schneider, C. Haas, M. Akmal, S. Söhner, T. Schaal, Dr. Ing. h.c. F. Porsche AG, Weissach;

W. Stork, KIT, Karlsruhe

Akzeptanz von Registrierungsfehlern in einem Augmented Reality Head-up-Display . . . . . 129

V. Sadovitch, M. Wittkämper, Volkswagen AG, Wolfsburg

Langnachleuchtende Luminophore zur Anwendung im Bereich des

hochautomatisierten Fahrens . . . . . . . . . . . . . . . . . . . . . . . 145

J. Arras, Karlsruher Institut für Technologie (KIT), Karlsruhe

\section{Visualisierung und Simulation}

Messmethoden zur Bewertung dynamischer Blendsituationen. . . . . . . . . . . . . . . 147

M. Helmer, C. Neumann, Karlsruher Institut für Technologie (KIT),

Lichttechnisches Institut (LTI)

Durchgängige simulative Evaluierung von Lichtfunktionen . . . . . . . . . . . . . . . . 159

5. Strebel, Dr. Ing. h.c. F. Porsche AG, Weissach;

C. Neumann, KIT - Lichttechnisches Institut, Karlsruhe

Systemverifikation hochkomplexer Lichtquellen - Ein notwendiger Schritt auf dem Weg zu hochauflösenden Scheinwerfersystemen. . . . . . . . . . . . . . . . . . . . . . 173

B. Kleinert, M. Marutzky, T. Uekermann, S. Bogdanow, IAV GmbH, Gifhorn;

T. Liebetrau, S. Stögner, Infineon Technologies AG, Neubiberg 


\section{Kommunikation und Wirkung von Licht}

Animierte Leuchten-Funktionen: "Spielereien" als Vorreiter visueller Car-to-X Kommunikation.

M. Ritter, C. Adikari, M. Richter, Automotive Lighting, Reutlingen

Realisierungsmöglichkeiten für hochaufgelöste Umfeldprojektionen im Automobil -

Bewertung unterschiedlicher Technologien . . .

C. Bremer, BMW AG, München, Karlsruher Institut für Technologie, Karlsruhe;

C. Neumann, Karlsruher Institut für Technologie, Karlsruhe;

B. Lewerich, BMW AG, München

Erster Ansatz zur abbildenden Umsetzung einer hochauflösenden Signalleuchte für automatisiertes Fahren . . . . . . . . . . . . . . . . . . . . 207

J.-H. Willrodt, Institut für Automobile Lichttechnik und Mechatronik (L-LAB), Lippstadt

A. Klarius, HELLA GmbH \& Co. KGaA, Lippstadt;

J. Wallaschek, Institut für Dynamik und Schwingungen (IDS) der Leibniz Universität Hannover

Auswirkungen von Mesostrukturen im Fahrzeuginnenraum auf die

Reflexionseigenschaften ... . . . . . . . . . . . . . . . . . . . . . . . . . 219

M. Schöneich, Daimler AG, Sindelfingen;

C. Neumann, Karlsruher Institut für Technologie (KIT), Lichttechnisches Institut Karlsruhe;

R. Lachmayer, Leibniz Universität Hannover, Institut für Produktentwicklung und Gerätebau

\section{$>$ Signalfunktion}

Progressive LED-Blade-Technologie für eindrucksvolle Fahrzeugleuchten -

Eindrucksvoll, robust und kostengünstig .

T. Hornung, A. Ebenbichler, M. Vollmer, odelo GimbH, Stuttgart

Diffraktive Diffusoroptiken als Ersatz für Volumenstreuer . . . . . . . . . . . . . . . . . . 245

M. Mügge, HELLA GmbH \& Co. KGaA, Lippstadt

Innovationspotenzial in der automobilen Lichttechnik - Vergleich Exterior vs. Interior . . . . . 265

D. Decker, Senior Consultant, PGUB Management Consultants GmbH, Bickenbach 